\title{
The Prognostic Significance of Preoperative Platelet-to-Lymphocyte and Neutrophil-to- Lymphocyte Ratios in Patients Operated for Non-Small Cell Lung Cancer
}

\author{
Mariusz Łochowski' \\ Justyna Chałubińska- \\ Fendler $\mathbb{( D}^{2}$ \\ Izabela Zawadzka ${ }^{3}$ \\ Barbara Łochowska ${ }^{4}$ \\ Marek Rębowski' \\ Daniel Brzeziński \\ Józef Kozak' \\ 'Clinic of Thoracic Surgery and \\ Respiratory Rehabilitation, Medical \\ University of Lodz, Lodz, Poland; \\ ${ }^{2}$ Department of Radiotherapy, Military \\ Institute of Medicine, Warsaw, Poland; \\ 3"Synevo" Medical Laboratory, Regional \\ Multi-Specialist Center for Oncology and \\ Traumatology of the Nicolaus Copernicus \\ Memorial Hospital in Lodz, Lodz, Poland; \\ ${ }^{4}$ Department of Radiotherapy and \\ General Oncology, Regional Multi- \\ Specialist Center for Oncology and \\ Traumatology of the Nicolaus Copernicus \\ Memorial Hospital in Lodz, Lodz, Poland
}

Correspondence: Mariusz Łochowski Email marilo@op.pl
Introduction:: The aim of the study was to determine the prognostic significance of PLR and NLR ratios in patients operated due to non-small cell lung cancer.

Material: The study group consisted of 532 (174 women, 358 men) patients with non-small cell lung cancer (NSCLC) staged IA-IIIA. The mean age was 63.6 years (range 36 to 84 years). Together with platelet/lymphocyte ratio (PLR) and neutrophil/lymphocyte ratio (NLR), the following factors were included in the statistical analysis: age, sex, smoking history, the number of leukocytes, neutrophils, and platelets, histopathology, T-stage, N-stage, concomitant diseases according to the Charlson Comorbidity Index (CCI), type of operation, adjuvant chemotherapy, and overall survival. Results: Univariate analysis showed an association between the value of PLR and NLR and the length of survival. Multivariate analysis found that the stage of advancement of the neoplastic disease $(\mathrm{p}=0.00003)$, adjuvant chemotherapy $(\mathrm{p}=0.009)$, CCI $>4(0.00008)$, and PLR $>144(\mathrm{p}=0.001)$ were negative prognostic factors for survival $>2$ years; however, this effect diminishes in patients surviving more than 5 years.

Conclusion: PLR might serve as a prognostic factor in patients affected by NSCLC with expected two-year overall survival.

Keywords: carcinoma, lymphocyte count, neutrophil count, non-small-cell, platelet count, ratio

\section{Introduction}

Lung cancer poses a serious threat, resulting in more than 1.6 million deaths worldwide annually. ${ }^{1}$ Unfortunately, it is most often diagnosed when it is already locally advanced, and about one-fifth of the total number of cases of non-small cell lung cancer (NSCLC) can qualify for surgical treatment. Despite intensive treatment, the 5-year survival expectancy of those patients does not exceed $18 \%$.

The most important prognostic factors are undoubtedly the histopathological type of cancer and its stage, ie lymph node involvement and presence of distant metastases. ${ }^{2}$ The majority of ongoing studies are focused on the search for other factors that can be assessed at disease onset to predict the prognosis and qualify the patient for appropriate treatment. Such studies are typically performed on a large scale, including huge panels of laboratory parameters including protein and genetic biomarkers which might indicate a biological response to oncogenesis ${ }^{3,4}$ This biological response is accompanied by a developing inflammatory process that can be induced by serum proteins and nucleic acid products released by the tumor, eg TNF-alpha, free fractions of microRNA or free circulating 
DNA. Many of these molecules are undergoing testing as possible prognostic markers; however, their precise accuracy and specificity remain unknown and they are routinely used. ${ }^{3}$ Nevertheless, it is possible to detect inflammatory processes with simple, easily available everyday tests, such as the standard complete blood count test and its calculated derivatives. ${ }^{4,5}$ In addition, the platelet-to-lymphocyte ratio (PLR) and the neutrophil-to-lymphocyte ratio (NLR) are promising indices whose prognostic value has been discussed in cases of various malignancies. $^{2,4}$

The aim of the present study is to evaluate the prognostic value of PLR and NLR in patients receiving surgical treatment due to NSCLC.

\section{Materials and Methods}

\section{The Patient Group}

The study included 532 patients surgically treated for stage IA-IIIA NSCLC in the period 2007 to 2014. Patients with operable small cell carcinoma and carcinoid were excluded from the study. Before the operation, all the patients had had to undergo a PET examination. Patients with N2 were qualified for an operation only if the metastases involved a single node and the node was not conglomerated.

The laboratory tests necessary for hospitalization were performed with a Sysmex XN 2000 flow cytometer (Sysmex Europe GmbH, Norderstedt, Germany). PLR, ie the ratio of the absolute number of platelets to the absolute number of lymphocytes, and NLR, the ratio of the absolute number of neutrophils to the absolute number of lymphocytes, were calculated in all analyzed patients.

\section{Patient Characteristics}

The study included 174 women and 358 men, aged 36-84 years (mean age: 63.6 years). Fifty-five percent of the patients indicated nicotine addiction. Imaging examinations revealed involvement of the right lung in 311 cases and involvement of the left lung in 221 cases. Squamous cell carcinoma (269 cases), adenocarcinoma (204 cases), large cell carcinoma (43 cases), and adenosquamous carcinoma (16 cases) were indications for surgical treatment. Most patients (375 cases) scored four to six points on the Charlson Comorbidity Index (CCI), while 100 patients scored 2 or 3 points (Table 1).

The study was granted approval by the Bioethics Committee of the Medical University of Lodz (consent
Table I Patient Characteristics and Results of Surgical Treatment with Survival Time

\begin{tabular}{|c|c|c|c|c|}
\hline Parameter & $\mathbf{n}$ & $\%$ & $\begin{array}{c}\text { Median of } \\
\text { Survival } \\
\text { [Years] }\end{array}$ & $\mathbf{p}$ \\
\hline \multicolumn{5}{|l|}{ Sex } \\
\hline $\mathrm{F}$ & 174 & 33 & 3.42 & 0.001 \\
\hline M & 358 & 67 & 2.46 & \\
\hline \multicolumn{5}{|l|}{ Nicotinism } \\
\hline Yes & 295 & 55 & 2.36 & 0.006 \\
\hline No & 237 & 45 & 3.09 & \\
\hline \multicolumn{5}{|l|}{ Charlson Comorbidity Index } \\
\hline 2 & 20 & 4 & & \multirow{8}{*}{0.0000} \\
\hline 3 & 80 & 15 & & \\
\hline 4 & $|4|$ & 27 & 3.76 & \\
\hline 5 & 126 & 24 & 2.13 & \\
\hline 6 & 108 & 20 & & \\
\hline 7 & 43 & 8 & & \\
\hline 8 & 12 & 2 & & \\
\hline 9 & 2 & 0.004 & & \\
\hline \multicolumn{5}{|l|}{ Localization - lung } \\
\hline Right & 312 & 59 & 2.90 & 0.04 \\
\hline Left & 220 & 41 & 2.42 & \\
\hline \multicolumn{5}{|l|}{ Localization - lobe } \\
\hline Lobe right upper & 178 & 34 & 3.01 & 0.01 \\
\hline Middle & 16 & 3 & 3.71 & \\
\hline Right low & 118 & 22 & 2.62 & \\
\hline Left upper & 119 & 22 & 2.89 & \\
\hline Left lower & 101 & 19 & 1.74 & \\
\hline \multicolumn{5}{|l|}{ Stage of the disease } \\
\hline $\mathrm{T}|\mathrm{A}|$ & 3 & 1 & 7.37 & 0.0000 \\
\hline T IA2 & 40 & 8 & 7.48 & \\
\hline T IA3 & 39 & 8 & 5.39 & \\
\hline $\mathrm{T} I \mathrm{~B}$ & 138 & 25 & 3.27 & \\
\hline T IIA & 8 & 2 & 0.78 & \\
\hline $\mathrm{T} I \mathrm{II}$ & 172 & 32 & 2.08 & \\
\hline $\mathrm{T} I I I \mathrm{~A}$ & 132 & 24 & 1.81 & \\
\hline \multicolumn{5}{|l|}{ Diagnosis } \\
\hline Squamous cell carcinoma & 269 & 50 & 2.84 & 0.7 \\
\hline Adenocarcinoma & 204 & 38 & 2.39 & \\
\hline Large cell carcinoma & 42 & 8 & 2.83 & \\
\hline Mixed type carcinoma & 16 & 4 & 2.14 & \\
\hline \multicolumn{5}{|l|}{ Surgical treatment } \\
\hline Lobectomy & 400 & 75 & 2.73 & 0.004 \\
\hline Bilobectomy & 37 & 7 & 3.49 & \\
\hline Pneumonectomy & 94 & 18 & 1.67 & \\
\hline
\end{tabular}


No. RNN/83/19/KE). Due to the retrospective character of the study, individual consent was not required from the patients for the review of medical records. The authors conformed to the principles of the Helsinki Declaration and kept the personal data of the participants confidential.

\section{Treatment Method}

All patients underwent typical anatomical resections, ie lobectomies, bilobectomies, and pneumonectomies. The operations were performed under general anesthesia, through the anterolateral approach with the use of a double-lumen tube. The surgeries involved the resection of at least six N1 and N2 lymph node groups according to the Classification of the Japan Lung Cancer Society. ${ }^{6}$ The tumor stage was identified with the use of the eighth edition of TNM (UICC from 2017). ${ }^{7}$ The resected lymph nodes were identified according to the Naruke map. ${ }^{8}$

\section{Statistical Analysis}

To determine the potential prognostic values of PLR and NLR parameters, the following factors were included in the analysis: patient age, sex, smoking history, the number of leukocytes, neutrophils, and platelets, histopathology, TNM staging, the Charlson Comorbidity Index, type of surgery, adjuvant chemotherapy, and overall patient survival.

The distribution of continuous variables was tested with the Shapiro-Wilk test. Continuous or ordinal variables without a normal distribution were presented as medians and lower and upper quartiles (25-75\%), and further analyses were made with the use of nonparametric tests. Normally distributed variables were presented as percentages and numbers. Pairs were compared using the Mann-Whitney $U$-test. Groups of more than two were compared with the Kruskal-Wallis ANOVA test, with the post hoc Dunn-Bonferroni analysis, if indicated. Nominal variables were analyzed using the chi-square test, and Spearman Rank for correlation.

Survival analysis was performed using the KaplanMeier test. Univariate analysis (UA) was performed using the Log rank test for nominal variables, and the Cox proportional hazard (CPH) model for continuous variables. For multivariate analysis, a $\mathrm{CPH}$ model was constructed including all factors presented as significant during UA; both backward and forward stepwise analyses were performed.

Receiver Operating Characteristic (ROC) curves were created based on these findings, and the optimal combination of sensitivity and specificity of the studied parameters was determined by means of the Youden method and calculated cut-off points.

\section{Results}

\section{Surgical Treatment and Postoperative Stages of Advancement}

The most commonly performed surgery was lobectomy (400 cases). Pneumonectomy was performed in 94 cases (18\%), whereas bilobectomy was administered in 37 cases. A postoperative histopathological analysis revealed $\mathrm{R} 0$ resection in all patients. Stage I was noted in $220(41 \%)$ cases, stage II in 180 (34\%) cases, and stage III in 132 $(25 \%)$ cases. Three patients died during postoperative hospitalization and another five within 30 days following surgery. The deaths were caused by myocardial infarct, cerebral stroke, and gastric ulcer perforation. Postoperative complications were observed in 112 cases $(21 \%)$; these included prolonged air leak, which occurred most frequently (59 cases), atelectasis (20 cases) requiring interventional bronchoscopy and postoperative anemia (19 cases), which required blood transfusion. Ten patients had to be treated for postsurgical arrhythmia. In addition, 228 patients received adjuvant chemotherapy based on combined administration of cisplatin with either vinorelbine or gemcitabine. When cisplatin was contraindicated, carboplatin was used. A total of 76 patients did not receive adjuvant treatment due to contraindication or lack of consent.

\section{Overall Survival (OS) of Patients}

The mean OS was 50 months, with $76 \%$ of patients surviving 1 year, $57 \%$ surviving 2 years, and $46 \%$ surviving 3 years. In the studied group of patients, the mean OS was 50 months; one-year OS was noted in $76 \%$ of patients, two-year OS in 57\%, and three-year OS in $46 \%$.

The survival analysis revealed that stages $\mathrm{T} 3$ and $\mathrm{T} 4$ and stages $\mathrm{N} 1$ and $\mathrm{N} 2$ most significantly contributed to shorter survival $(\mathrm{p}=0.0009$ and $\mathrm{p}=0.0000$, respectively). The mean survival periods were 4.605 for stage $\mathrm{T} 1$ and $\mathrm{T} 2,3.934$ for $\mathrm{T} 3$ and $\mathrm{T} 4,4.80$ for N0, 3.66 for N1, and 2.76 for $\mathrm{N} 2(\mathrm{p}=0.0000)$. Sex and nicotine addiction most significantly affected patient survival $(\mathrm{p}=0.00117$ and $\mathrm{p}=0.006$, respectively). The mean survival periods were 4.87 years for women and 3.91 years for men and 3.88 years for nicotine smokers and 4.63 years for nonsmokers. Longer OS was observed after lobectomies and bilobectomies (respectively: 4.42 years and 4.51 years) 
A

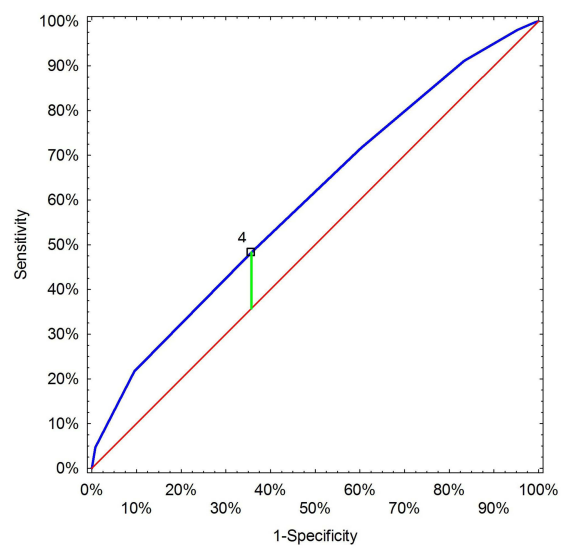

B

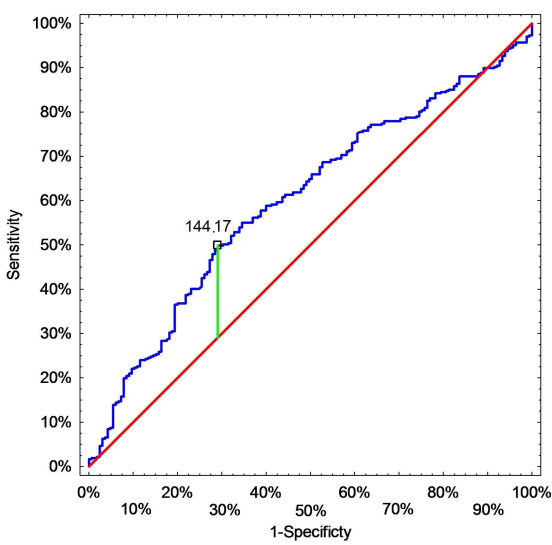

C

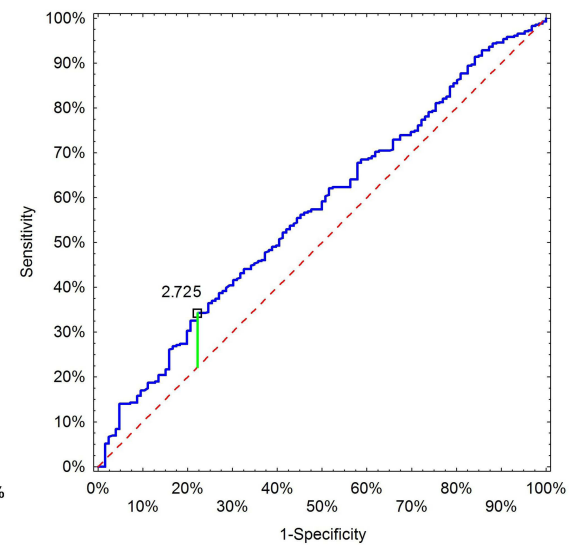

Figure I Youden index with receiver-operating characteristic (ROC) curves. (A) Charlson comorbidity index (CCl). (B) Platelet to lymphocyte ratio. (C) Neutrophil to lymphocyte ratio.

than after pneumonectomies ( 3.23 years; $\mathrm{p}=0.0046)$. To a lesser extent, localization of the tumor in the right upper and middle lung lobe resulted in better survival (3.01 years and 3.71 years; $\mathrm{p}=0.01$ ). No significant relationship was observed between tumor type and patient OS $(p=0.7)$. The CCI value was found to be 4 , based on the ROC value $(\mathrm{p}=0.005)$. Patients with $\mathrm{CCI}>4$ demonstrated significantly shorter OS ( 3.55 versus $5.02 ; \mathrm{p}=0.0000)$, as can be seen in Table 1 .

\section{PLR and NLR as Prognostic Factors}

The obtained AUCs were 0.567 for PLR (95\% CI: 0.509$0.624 ; \mathrm{p}=0.0238)$ and 0.587 for NLR (95\% CI: $0.531-0.642$; $\mathrm{p}=0.0024)$.. The cutoff point values for PLR and NLR were 144.017 and 2.725 , respectively (Figure 1).

Univariate analysis indicated that NLR and PLR had a significant effect on patient OS, as did CCI and application of adjuvant chemotherapy (Table 2 and Figure 2).

A multivariate analysis of patients treated for NSCLC with OS $>2$ years revealed that adjuvant chemotherapy; $\mathrm{p}=0.009, \mathrm{CCI}>4 ; \mathrm{p}=0.00008$ and PLR $>144, \mathrm{p}=0.001 \mathrm{can}$ be considered independent prognostic factors of advanced stage of lung cancer. However, the prognostic value of these parameters decreases for OS greater than 5 years (Table 3). Figure 3 presents the Kaplan-Meier curves of OS $>2$ years in the multivariate model.

\section{Discussion}

The chronic inflammatory process accompanying neoplasia can be easily confirmed by simple blood tests. The most frequently observed abnormalities include quantitative changes in simple blood tests, such as leukocytosis, neutrophilia, thrombocytopenia, and lymphocytopenia. ${ }^{5}$ They occur during the growth and lysis of the tumor. Immunoregulatory cytokines secreted by inflammation facilitate the recruitment of tumor-associated neutrophils, causing disease progression and increasing the risk of distant metastasis; platelets also appear to have a similar role. ${ }^{5}$ In contrast, lymphocytes display completely different behavior and are believed to have anticancer activity; indeed, lymphocytosis itself is considered a favorable prognostic factor. ${ }^{9}$

These abnormalities demonstrate a mutual relationship, as reflected in the PLR and NLR parameters. ${ }^{5,9}$ Although

Table 2 Results of Studied Parameters and Univariate Analysis in Patients Operated on Due to NSCLC

\begin{tabular}{|l|c|c|c|c|c|}
\hline Parameter & Median & Range & HR & 95\% CI & P \\
\hline Neutrophils $\left[\times 10^{3}\right]$ & 5.8 & $1.4-31.0$ & 1.03 & $1.01-1.04$ & 0.02 \\
Lymphocytes $\left[\times 10^{3}\right]$ & 1.9 & $0.5-9.3$ & 0.84 & $0.72-0.97$ & 0.02 \\
Platelets $\left[\times 10^{3}\right]$ & 262.5 & $30.0-674.0$ & 1.00 & $1.00-1.00$ & 0.16 \\
NLR & 2.7 & $0.5-12.3$ & 1.05 & $1.02-1.09$ & 0.00 \\
PLR & 144.0 & $17.6-570$ & 1.00 & $1.00-1.00$ & 0.00 \\
CCl & 4.2 & $2-9$ & 1.21 & $1.12-1.30$ & 0.00 \\
\hline
\end{tabular}




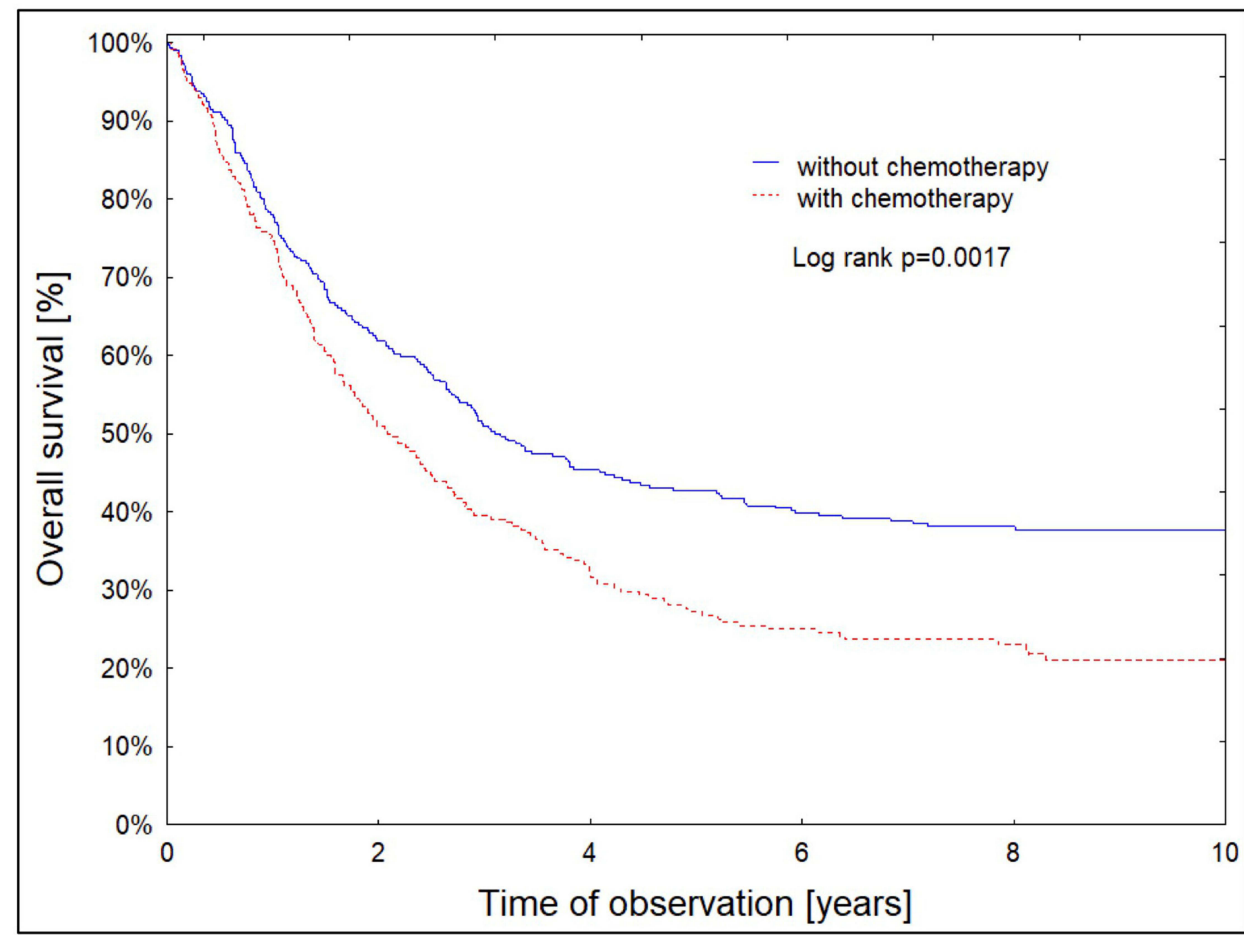

Medi Mean OS SD OS [y] $\mathrm{N}$-non $\mathrm{N} \quad \mathrm{N}$ total

an $[\mathrm{y}] \quad$ censored censored

OS

[y]

$\begin{array}{lllllll}\begin{array}{l}\text { No } \\ \text { chemotherapy }\end{array} & 3,12 & 4,72 & 3,85 & 189 & 115 & 304 \\ \text { Chemotherapy } & 2,11 & 3,55 & 3,36 & 178 & 50 & 228 \\ \text { Total } & 2,68 & 4,22 & 3,69 & 367 & 165 & 532\end{array}$

Figure 2 Impact of adjuvant chemotherapy on patient survival.

their prognostic role of these parameters in NSCLC has clinical practice. ${ }^{10,11}$ Nevertheless, as the test is widely been widely discussed in meta-analyses, the authors available and easy to perform, and the results are obtained recommend caution in their interpretation and use in quickly afterwards, it was included in the present study.

Table 3 Multivariate Analysis in Patients Operated on Due to NSCLC

\begin{tabular}{|c|c|c|c|c|c|c|}
\hline \multirow[t]{2}{*}{ Parameter } & \multicolumn{3}{|c|}{ Model of OS $>2$ Years } & \multicolumn{3}{|c|}{ Model of OS $>5$ Years } \\
\hline & HR & $95 \% \mathrm{Cl}$ & $\mathbf{p}$ & HR & $95 \% \mathrm{Cl}$ & $\mathbf{p}$ \\
\hline Neutrofile $\left[\times 10^{3}\right]$ & 1.145 & $0.986-1.331$ & 0.075 & 1.032 & $0.882-1.207$ & 0.686 \\
\hline Limfocyty $\left[\times 10^{3}\right]$ & 1.137 & $0.643-2.012$ & 0.656 & 0.965 & $0.550-1.694$ & 0.903 \\
\hline Platelets $\left[\times 10^{3}\right]$ & 0.997 & $0.992-1.002$ & 0.366 & 0.996 & $0.991-1.001$ & 0.124 \\
\hline NLR $>2.725$ & 0.947 & $0.7|3|-1.395$ & 0.988 & 0.939 & $0.742-1.427$ & 0.606 \\
\hline PLR $>144$ & 1.004 & $1.00 \mathrm{I}-1.006$ & 0.001 & 1.005 & $0.997-1.014$ & 0.177 \\
\hline $\mathrm{CCl}>4$ & 1.319 & $1.149-1.514$ & 0.00008 & 0.725 & $0.628-0.838$ & 0.00001 \\
\hline Stages: & 1.754 & $1.086-1.833$ & 0.00003 & 1.154 & $1.047-1.273$ & 0.0002 \\
\hline Chemotherapy & 1.163 & $0.916-1.477$ & 0.009 & 1.281 & $0.906-1.811$ & 0.498 \\
\hline Sex & 0.912 & $0.590-1.407$ & 0.677 & 1.233 & $0.804-1.893$ & 0.335 \\
\hline Smoking & 1.345 & $0.911-1.986$ & 0.135 & 1.257 & $0.850-1.858$ & $0.25 I$ \\
\hline
\end{tabular}

Note: Statistically significant values are printed as bold. 


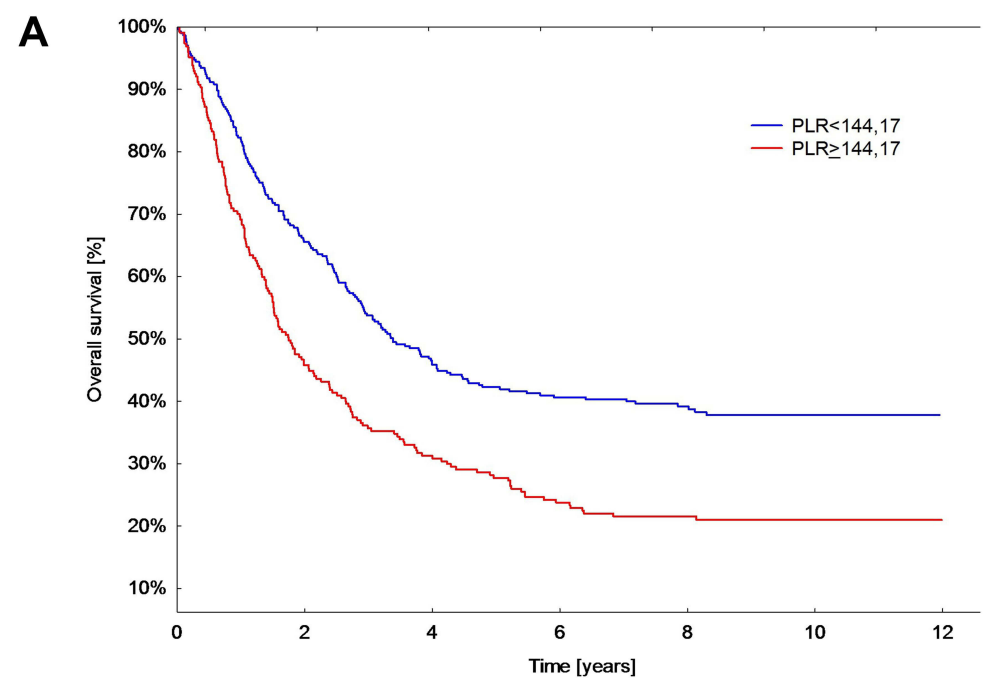

B

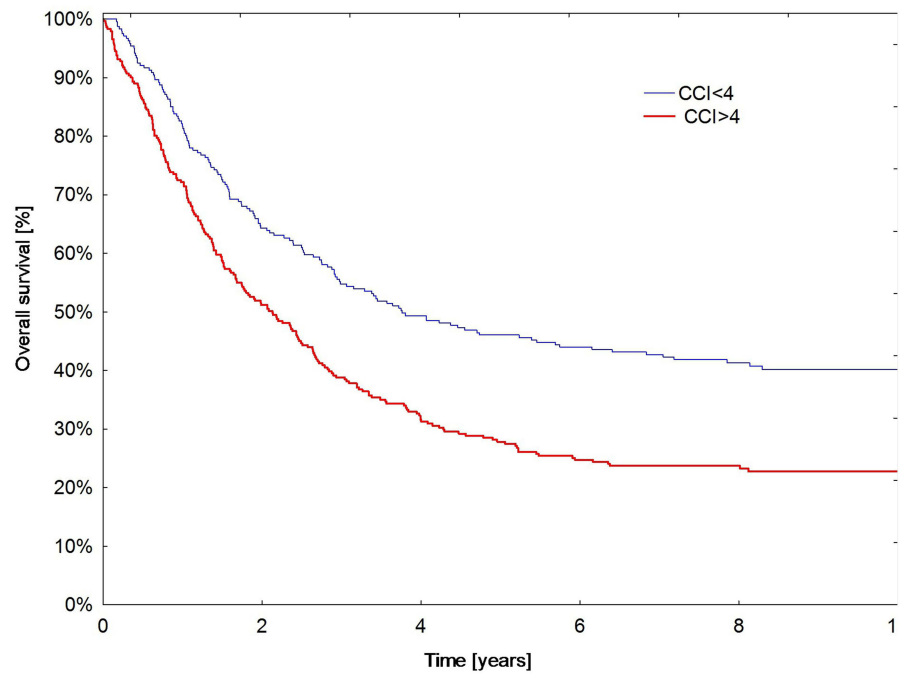

C

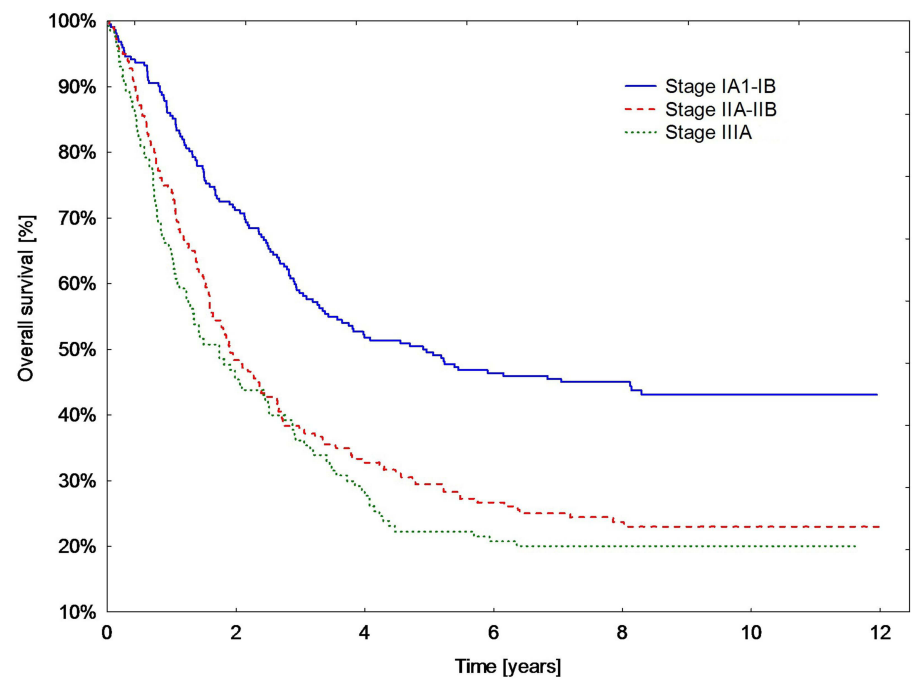

Figure 3 Kaplan-Meier curves of survival of patients operated on due to non-small cell lung cancer. (A) Platelet to lymphocyte ratio. (B) Charlson comorbidity index (CCl). (C) Stage of disease. 
Most of the reports on NLR and PLR parameters are from eastern Asia. ${ }^{9-12}$ The authors of this study wanted to investigate the behavior of these indicators in the population of an Eastern European country.

Our observations indicate that increased values of PLR and NLR, assessed preoperatively, are related to the OS of NSCLC patients after radical surgical treatment. Similar preoperative prognostic findings have been obtained previously, ${ }^{12}$ as well as in NSCLC patients treated with Nivolumab. ${ }^{13}$

Our analysis showed that PLR demonstrates a greater preoperative prognostic value than NLR. The survival time of patients over 2 years (OS $>2$ ) was significantly associated with a worse prognosis $(p=0.001)$ for PLR $>144$; no such association was noted for NLR. However, no prognostic value was observed for either of the parameters in the analysis of OS $>5$ years. Similarly, Huang et al report that PLR values $>122$ significantly affect the OS of patients, and this parameter shows a higher prognostic value than NLR. ${ }^{14}$ Lan et al observed the highest prognostic value of PLR, ie $>148$, for one-year survival. ${ }^{12}$ A meta-analysis by Qiang et al highlights the value of PLR as a cheap and easily available negative prognostic marker for DFS and OS in patients diagnosed with NSCLC. ${ }^{10}$

Our own multivariate analysis showed that PLR $>144$, $\mathrm{CCI}>4$, advancement stages IIA-B and IIIA and application of adjuvant chemotherapy are all independent prognostic factors contributing to a worse prognosis in patients surgically treated for NSCLC. Lee et al obtained similar results, noting that age, male sex, PLR $>180$, TNM stages II and III and postoperative radiotherapy are prognostic disadvantages for NSCLC patients. ${ }^{15} \mathrm{Xu}$ et al report a relationship between PLR $>135$ and $\mathrm{T}$ stage, ${ }^{2}$ while Toda et al indicate that PLR is an unfavourable prognostic factor in NSCLC patients who were treated surgically and received adjuvant chemotherapy. ${ }^{16}$ This latter observation is consistent with our present findings, which indicate that PLR values are related to TNM stages II and III and adjuvant chemotherapy.

Our work has several limitations. This study was retrospective in character, and it was performed in a single center for which only patients with operable NSCLC were included. As such, the selection of patients had an influence on the obtained results. Secondly, the postoperative staging in the study group revealed stages IIB and IIIA; therefore, these patients received complementary treatment, which also had an impact on the analyzed survival time. Thirdly, the authors did not have any data on the molecular profile of the patients, which to some extent was a limitation of this study.

\section{Conclusions}

1. In NSCLC patients, elevated PLR values appear to be an independent prognostic factor, in addition to advanced stage and coexistent diseases defined by the Charlson Comorbidity Index.

2. Further prospective multi-center research should be carried out on larger groups of patients before inflammatory markers can be confidently interpreted as prognostic markers in lung cancer and applied in clinical practice.

\section{Abbreviations}

AUC, area under curve; CCI, Charlson Comorbidity Index; $\mathrm{CPH}$, cox proportional hazard; CT, computed tomography; EBUS, endobronchial ultrasound; NLR, neutrophil-to-lymphocyte ratio; NSCLC, non-small cell lung cancer; OS, overall survival; PET-CT, positron-emission tomography with computed tomography; PLR, platelet-tolymphocyte ratio; ROC, receiver operating characteristic; TNM, tumor node metastasis classification; UA, univariate analysis; UICC, union of international cancer control.

\section{Disclosure}

All authors declare no conflicts of interest.

\section{References}

1. de Grot PM, Wu CC, Carter BW, Munden RF. The epidemiology of lung cancer. Transl Lung Cancer Res. 2018;7:220-233.

2. Xu F, Xu P, Cui W, et al. Neutrophil-to-lymphocyte and platelet-tolymphocyte ratios may aid and identifying patients with non-small cell lung cancer and predicting tumor-node-metastases stage. Oncol Lett. 2018;16:483-489.

3. Wei L, Wu W, Han L, Yu W, Du Y. A quantitative analysis of the potential biomarkers of non-small cell lung cancer by circulating cell-free DNA. Oncol Lett. 2018;16:4353-4360.

4. Sayan M, Kankoc A, Ozkan ND, et al. Simple peripheral blood cell parameters to predict prognosis in non-small cell lung cancer. Indian J Surg. 2020. doi:10.1007/s12262-020-02237-4

5. Sahin F, Aslan AF. Relationship between inflammatory and biological markers and lung cancer. J Clin Med. 2018;7. doi:10.3390/ jem7070160

6. Itazawa T, Tamaki Y, Komiyama T, et al. The Japan Lung Cancer Society-Japanese Society for Radiation Oncology consensus-based computed tomographic atlas for defining regional lymph node stations in radiotherapy for lung cancer. $J$ Radiat Resp. 2017;58:86-105. doi: $10.1093 / \mathrm{jrr} / \mathrm{rrw} 076$

7. Detterbeck FC. The eighth edition TNM stage classification for lung cancer: what does it mean on main street? J Thorac Cardiovasc Surg. 2018;155:356-359. 
8. El-Sherif AH, Lau CT, Carter BW, Wu CC. Staging lung cancer: regional lymph node classification. Radiol Clin North Am. 2018;56:399-409. doi:10.1016/j.rcl.2018.01.008

9. Wang L, Si H, Wang J, et al. Blood cell parameters as prognostic predictors of disease development for patients with advanced non-small cell lung cancer. Oncol Lett. 2020:20. doi:10.3892/ ol2020.11655

10. Quiang G, Liang C, Xiao F, et al. Prognostic significance platelet-tolymphocyte ratio in non-small-cell lung cancer: a meta-analysis. Onco Targets Ther. 2016;22:869-876. doi:10.2147/OTT.S96804

11. Yu Y, Qian L, Cui J. Value of neutrophil-to-lymphocyte ratio for predicting lung cancer prognosis: a meta-analysis of 7219 patients. MolClinOncol. 2017;7:498-506.

12. Lan H, Zhou L, Chi D, et al. Preoperative platelet to lymphocyte and neutrophil to lymphocyte ratios are independent prognostic factors for patients undergoing lung cancer radical surgery: a single institution cohort study. Oncotarget. 2017;8:35301-35310. doi:10.18632/ oncotarget.13312
13. Diem S, Schmid S, Kraft M, et al. Neutrophil -to-lymphocyte ratio (NLR) and Platelet-to-lymphocyte ratio (PLR) as a prognostic markers in patients in non-small cell lung cancer (NSCLC) treated with nivolumab. Lung Cancer. 2017;111:176-181. doi:10.1016/j. lungcan.2017.07.024

14. Huang Q, Diao P, Li C-L, et al. Preoperative platelet-lymphocyte ratio is a superior prognostic biomarker to the other systemic inflammatory response markers in non-small cell lung cancer. Medicine. 2020:99. doi:10.1097/DM0000000000018607

15. Lee MB, Rodrigeuz A, Mena G, et al. Platelet-to-lymphocyte ratio and use of NSIADs during perioperative period as prognostic indicators in patients with NSCLC undergoing surgery. Cancer Control. 2016;23:284-294. doi:10.1177/107327481602300312

16. Toda $\mathrm{M}$, Tsukioka $\mathrm{T}$, Isumi $\mathrm{N}$, et al. Platelet-to-lymphocyte ratio predict the prognosis of patient with non-small cell lung cancer treated with surgery and postoperative adjuvant chemotherapy. Thorac Cancer. 2018;9:112-119. doi:10.1111/1759-7714.12547

\section{Publish your work in this journal}

Cancer Management and Research is an international, peer-reviewed open access journal focusing on cancer research and the optimal use of preventative and integrated treatment interventions to achieve improved outcomes, enhanced survival and quality of life for the cancer patient.
The manuscript management system is completely online and includes a very quick and fair peer-review system, which is all easy to use. Visit http://www.dovepress.com/testimonials.php to read real quotes from published authors. 